\title{
Small- Scale Drilling Test Rig For Investigation of Axial Excitation On The Drilling Process
}

\author{
A. Austefjord, ${ }^{1}$ S. Blaylock, ${ }^{1}$ I. Forster, ${ }^{1 *}$, M. Sheehan, ${ }^{1}$ C. Wright ${ }^{1}$ \\ 1: National Oilwell Varco, Conroe, Texas, United States
}

\begin{abstract}
This paper describes the design, construction and operation of a small-scale drilling rig for the purpose of investigation of the effect of axial excitation on the drilling process. The rig is bench top in size and has been designed to drill small rock samples, whilst at the same allowing axial excitation to be induced into the drilling process. The rig has been designed to drill the rock without any drilling fluids - so allowing improved observation of the chip generation process. Additionally, the drilling weight on bit is applied via masses, so allowing greater representation of the dynamic behavior of the drilling process - i.e. capturing more natural frequencies. The results from the rig have been obtained over two frequency ranges - low frequency $(0-50 \mathrm{~Hz})$ and high frequency $(50-250 \mathrm{~Hz})$. Results show that improved rate of penetration is obtained with axial excitation - with low and high frequency optima occurring. These optima can be related to the behavior of the string in the two frequency ranges - in the low frequency range, the entire string acts in unison; whereas at high frequency, only the bit/rock system is active. As a result, it is concluded that for low frequency operation, only information about the drill string is required to optimize performance; whereas for high frequency operation, information about the bit/rock system is required to optimize performance. Observation of the chip generation process via high speed video has shown that during axial excitation, regular shaped bricks are ejected when compared with the typical wedge- shaped chips that are normally ejected during the drilling process. It is concluded that, during the axial excitation process, the chips are being ejected via a levering action, so allowing a more efficient and quicker process. MIT [1] provided background classes, project guidance and project review as part of an NOV/MIT advanced technology program. Larger scale lab tests and/or field tests are required to verify/validate these conclusions.
\end{abstract}

\section{Introduction}

With respect to National Oilwell Varco (NOV) and NOV customers, of particular interest has been vibrations that aid the drilling process. Current tools that meet this requirement are tools that aid the drilling process by inducing axial excitation into the drill bit - either at medium frequency or at low frequency. There are also potential future tools operating at higher and very low frequencies.

There have been many published works in this field however the most practical recent approaches, that have the greatest relevance for NOV, have been from the University of Aberdeen [2] and the University of Newfoundland [3].

Using the current axial excitation tools that are available as a basis for the study, on the small to medium scale, this has presented a low risk strategy to get a minimum viable product (MVP). The project was carried out on the as an innovation exercise, in order to determine if useful information could be obtained on such a fast track project.

\section{Test Rig Description}

The basic approach in the test rig design and its operation is that any modelling and small-scale tests should be concurrent and interactive and should cover all areas of the drill string - i.e. drilling bit (including bit/rock interaction), BHA and upper string.

It has been appropriate, given the broad subject matter, to cover one aspect of drilling vibration in isolation initially, but with a view to considering, and combining, other aspects in the future.

The small-scale tests and modelling have been initially discrete spring/mass based, with a view to develop into distributed mass/stiffness systems.

The modelling tools to achieve this have been math package based - MATHCAD/MATLAB for example [4] - and/or FEA based - i.e. ANSYS [5]. Lab tests have been initially in the vertical orientation, but could be developed into other orientations.

Rig test and mathematical modelling derived from testing can be used as a design tool for up- scale to full size down hole tools.

\footnotetext{
*Corresponding author: ian.forster@nov.com
} 
The test rig is shown in figure 1.

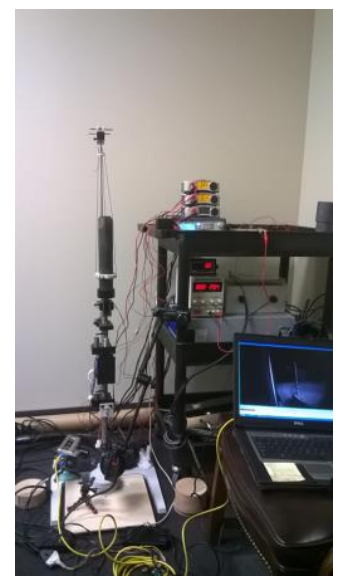

Figure 1 - Test rig

$\underline{\text { Small-scale rig - pros }}$

- Low cost.

- $\quad$ Fast build.

- $\quad$ Fail fast philosophy.

- Low risk.

- $\quad$ Small, light- weight - can be located virtually anywhere.

- $\quad$ Low voltage - minimal safety issues.

- Better to have it, than to be without it.

- $\quad$ Even if it fails, lessons will be learned - to the extent of achieving success at the next iteration.

- $\quad$ Allows NOV to be in a better position to ask the right questions - i.e. it helps to have a good idea of the answers first.

- Will allow any literature search to be more focused - i.e. will complement a literature search.

$\underline{\text { Small-scale rig - cons }}$

- Fails - see above.

The main design consideration has been the application of weight on bit via masses, rather than by force/actuator - so enabling the dynamic behavior of a typical drill string to be captured - i.e. more natural frequencies. Another design consideration is the use of standard, off the shaft readily available components - for example, using standard masonry bits. Dry cutting has also been adopted, to aid visualization of the chip generation process.

The small-scale approach has been adopted in other work [6].

\section{Test Rig Modelling}

Two independent modelling approaches were adopted running concurrently with the test rig design and operation: the first being a MATHCAD- based spring mass model approach; and the second ANSYS FEAbased spring model approach. The former allows more readily varied parameters and model adjustment; whereas the latter allows good visualization/animation of results.

The MATHCAD results in figure 2 show that the rig has two main natural frequencies - so justifying the mass based weight on bit application approach.

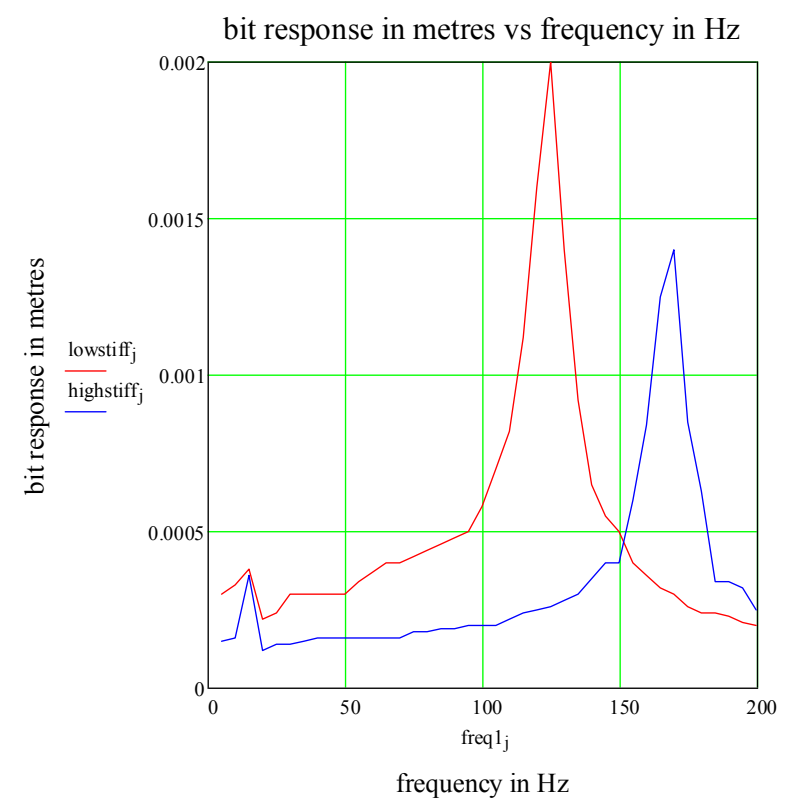

Figure 2 - MATHCAD rig model frequency response

The results are presented for two rock stiffness's - and indicate that the low frequency response is largely independent of rock stiffness - whereas the high frequency response is not. This high frequency result agrees with other published work (reference 1).

A further conclusion can be drawn from the ANSYS simulations - that the low frequency response is dependent on the drill string (with the drill string acting in unison); whereas the high frequency response is dependent on the bit rock system in isolation.

\section{Test Rig Results}

Test rig results presented are for soft rock and hard rock - sandstone and limestone. Two frequency ranges were considered - 0-50 Hz; and 50-250 Hz. High speed video was used to capture the chip generation process for the hard rock case.

\section{$\underline{\text { Soft Rock }}$}

For the soft rock results in figures 3 and 4, the largest percent gain in rate of penetration (ROP) was shown to coincide with the best low and high frequency rig

\footnotetext{
* Corresponding author: ian.forster@nov.com
} 
responses.

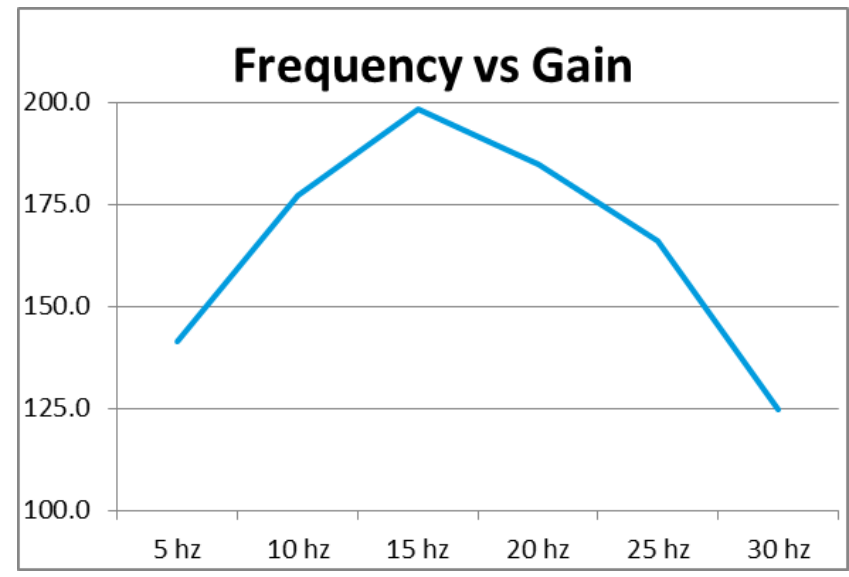

Figure 3 - Test rig low frequency percent ROP gains

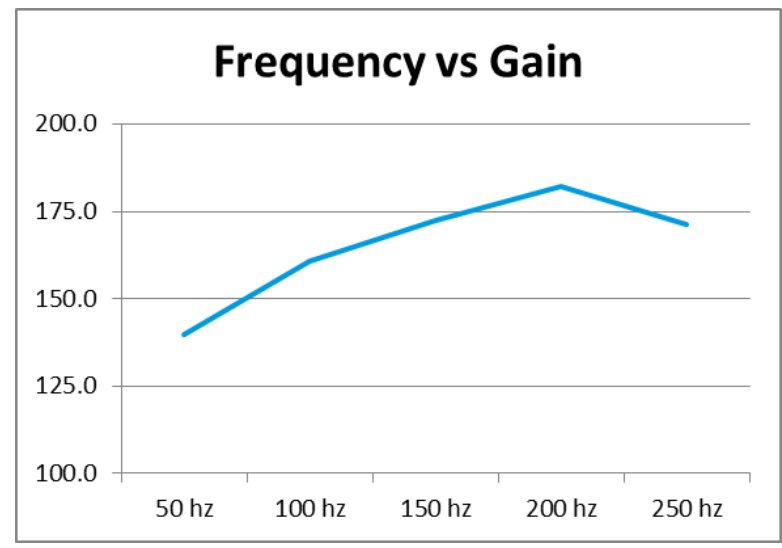

Figure 4 - Test rig high frequency percent ROP gains

\section{$\underline{\text { Hard Rock }}$}

With the harder rock at low frequency and an upgraded rig, the same peak ROP frequency was repeated - for small and larger bits; however, there was a very low frequency secondary peak - not see in the first results figures 5 and 6 . This latter feature may have been as a result of the excitation frequency synchronizing with the chip generation frequency, and may have not been apparent on the pre-upgrade rig - due to energy dissipation. Additionally, it could also be due, in part, to rig structural resonance (again, only apparent after rig upgrade). This justifies further investigation.

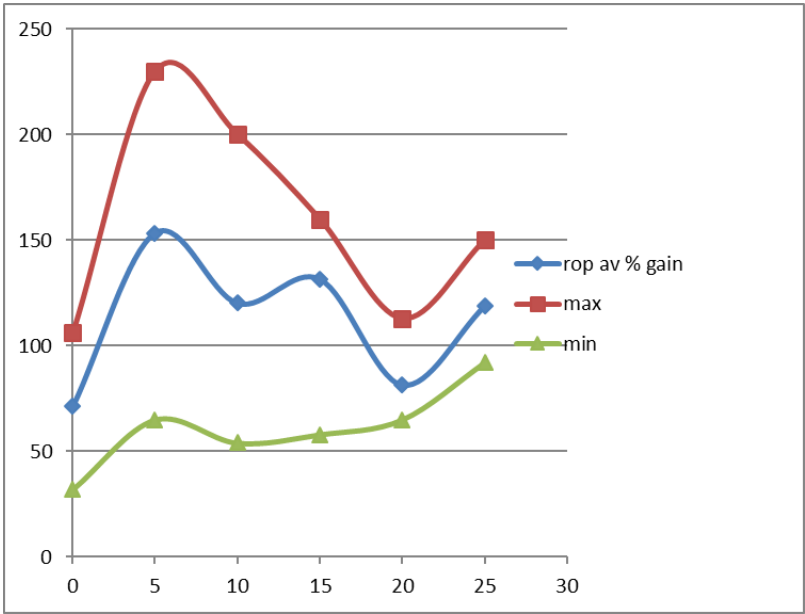

Figure 5 - Test rig low frequency small bit percent ROP gains

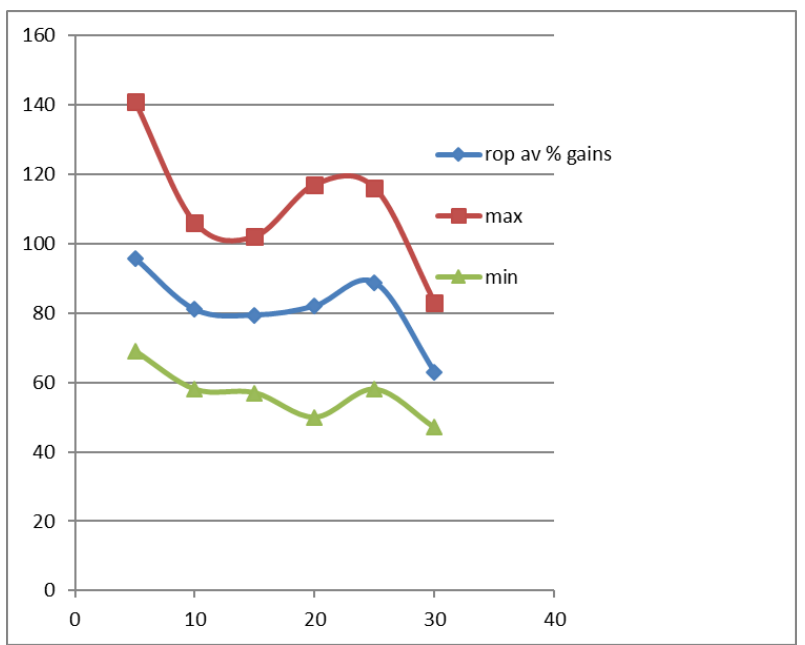

Figure 6 - Test rig low frequency large bit percent ROP gains

\section{High Speed Video}

High speed video test were conducted over a variety of frequencies - from $5 \mathrm{hz}$ up to $150 \mathrm{hz}$.

However, the $100 \mathrm{hz}$ result is presented here - as the migration of cuttings across the rock during the $100 \mathrm{~Hz}$ test, lended itself well to analysis of the debris field via microscope - figures 7-10. Note that the calutated normal drilling (non excitation) chip generation frequency based on depth of cut and rpm would be of the order of $100 \mathrm{~Hz}$. Observed chip generation frequency was of the order of the natural frequency of the string suggesting that this is an important driver.

Any issues with dry cutting were also investigated i.e. a back to back test was run with and without dust extraction applied - and no observable difference was noted in tersms of ROP. However, further invetigation is still justified.

From the microscope photos, the chip size and shape matched the expected size and shape based on excitation frequency, rpm and bit size.

\footnotetext{
* Corresponding author: ian.forster@nov.com
} 
A proposal for the chip generation process during axial excitation is given - suggesting a levering action is used to generate the chips - analogous to a jack hammer resulting in lower energy required to fail the rock (in bending and tension) - figure 11 .

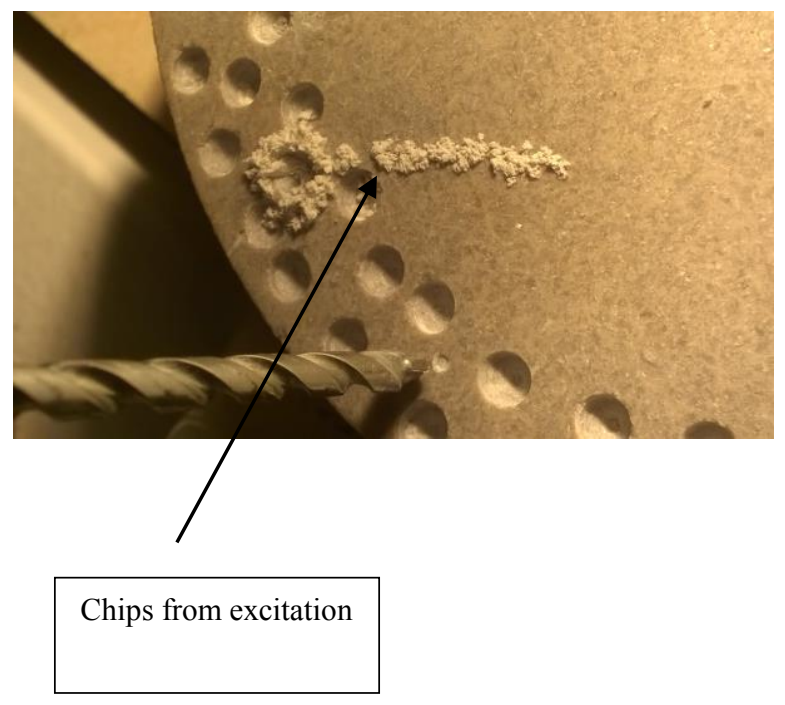

Figure 7 - debris field image

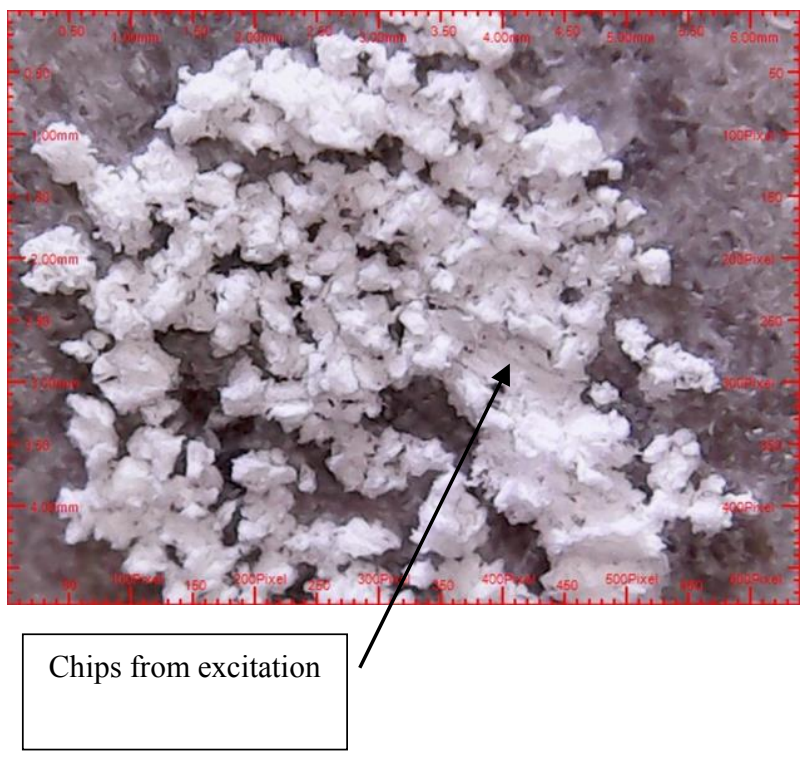

Figure 8-debris field microscope image

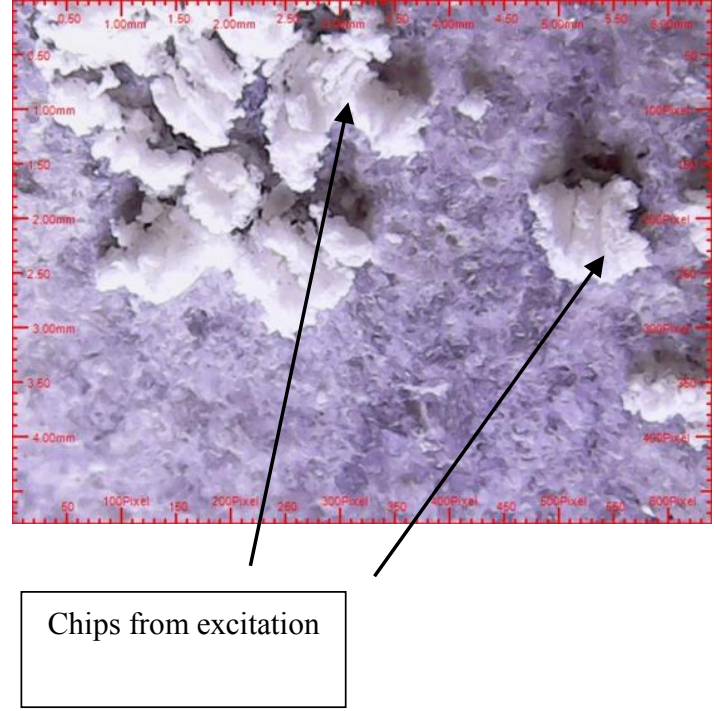

Figure 9- debris field microscope image
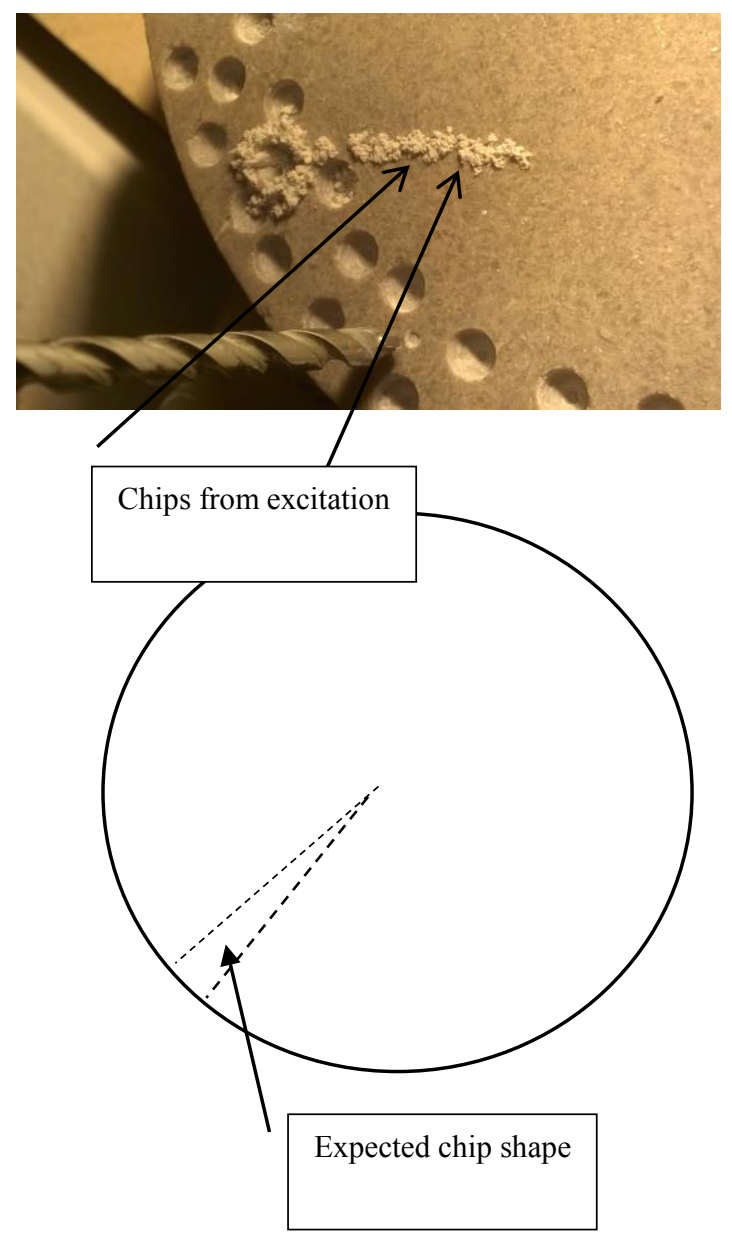

Figure 10 - expected chip shape

- Chip tensile failure and ejection - earlier due axial upward movement - chip size depends on frequency and rpm - chip is effectively levered out- smaller chip and small amount of 
material needing to be compressed/crushed chip size more controlled

Wob-Fa

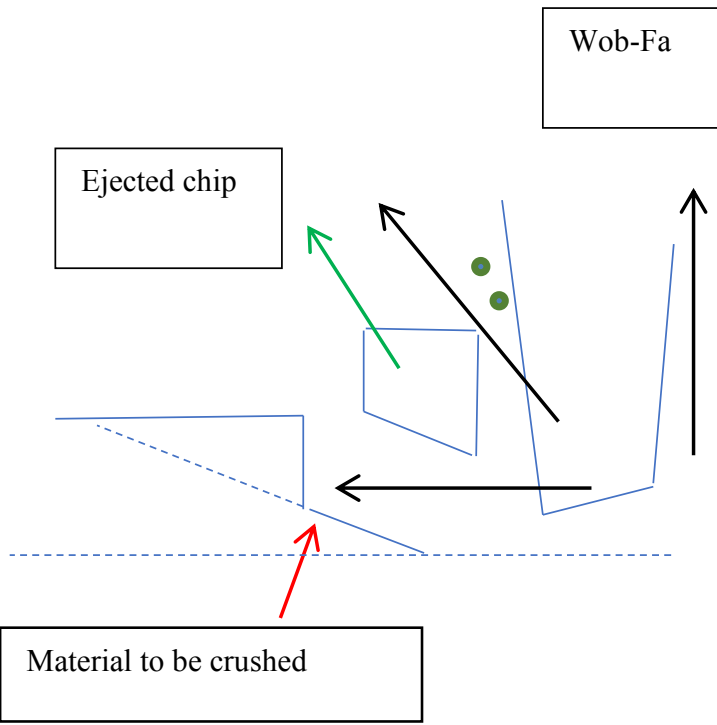

Note chip failure is mainly tensile - requiring less energy as the rock will have low tensile strength compared to compressive strength

Note also that lower frequency excitation will tend to form larger chips than at high frequency - at a given rpm

Figure 11 - excitation chip generation

\section{Results Summary}

1. Very low frequency benefits - possible synchronization with chip generation and/or rig structural resonance - quasistatic.

2. Low frequency peak ROP gain at $20-25 \mathrm{~Hz}$ coincident with string resonance.

3. High frequency peak ROP gain coincident with bit rock system resonance.

4. Larger bit tests - results variability offset.

5. Dry testing beneficial for visualization, effect on results checked but further investigation still required.

6. Mechanical specific energy (MSE) results mirrored ROP during excitation- but mitigated due to increased torque.

7. When electrical energy included in MSE formulation - increased by a factor of 9 .

\footnotetext{
* Corresponding author: ian.forster@nov.com
}

8. MSE suggests that a mechanical system would be preferable for excitation.

\section{Conclusions}

1. The small-scale rig approach has been validated.

2. Low and high frequency tools have been identified - and a possible very low frequency tool.

3. Low frequency tools are current NOV tools and therefore the results can be used for optimization and modelling development.

4. The chip generation results can feed into bit/cutter design.

5. High frequency tools are possible for the future.

\section{Further Work}

1. Further tests using hard rock

2. Further upgrades - higher data rate data acquisition for higher frequency tests.

3. Investigation of varying rock/support stiffness.

4. String modelling development with respect to NOV tools.

5. Further High- speed video of bit rock interaction

6. Higher frequency tests - up to $1 \mathrm{KHZ}$ and higher

7. Inclination tests

8. Further investigate the large variations in repeated, identical tests.

9. Very low frequency investigation with respect to synchronization with normal chip generation rate.

10. Cloned rigs to investigate specific phenomena in more detail - possibly at other establishments.

11. Investigation on any disadvantages of dry testing over wet testing.

\section{Acknowledgements}

1. ICOEV 2017

2. NOV

3. MIT

4. Project team members

\section{Glossary}

NOV - National Oilwell Varco

MIT - Massachusetts Institute of Technology

MSE - mechanical specific energy

RPM - revs per minute

ROP - rate of penetration

MVP - minimum viable product 
FEA - finite element analysis

WOB - weight on bit

$\mathrm{Fa}$ - axial excitation force

\section{References}

1 MIT/NOV - Advanced Technology Program - (2015-2016)

2 University of Aberdeen - publications relating work carried out on, and associated with, the Resonance Enhanced Drilling Project - (2009-2017)

3 University of Newfoundland - publications relating work carried out on, and associated with, axial excitation during drilling

4 MATHCAD - v14

5 ANSYS - v14.5

6 NOV - publications related to work carried out on, and associated with, the use of small-scale test rigs - (2009-2017)

7 G. Hareland et al., JCPT - paper number 2007-082 - Cutting Efficiency of a Single PDC Cutter on Hard Rock - (2007)

\footnotetext{
Corresponding author: ian.forster@nov.com
} 